\title{
Erratum to: Determination of plasma dibasic amino acids following trimethylsilyl-trifluoroacyl derivatization using gas chromatography-mass spectrometry
}

\author{
Hye-Ran Yoon
}

Published online: 31 May 2013

(C) The Pharmaceutical Society of Korea 2013

Erratum to: Arch. Pharm. Res. (2013) 36:366-373

DOI 10.1007/s12272-013-0038-1

Unfortunately the original published article contains an incomplete Acknowledgement section. Please find the full Acknowledgement section below.
Acknowledgments This research was supported by the Duksung Women's University Research Grants 2011, Seoul, South Korea. The author thanks to Hyunsu Jeong, Mirin Kang, and Heejoon Jeong for experiment and copyediting a manuscript.

The online version of the original article can be found under doi: 10.1007/s12272-013-0038-1.

\section{H.-R. Yoon $(\bowtie)$}

Department of Biomedical \& Pharmaceutical Analyses, College

of Pharmacy, Duksung Women's University, 33 Samyangro

144-gil, Seoul 132-714, South Korea

e-mail: hyeran11@duksung.ac.kr 